\title{
Organoleptic Properties and Acceptability of Fresh Beverages Using Wheat Grass Juice with some Fruit and Vegetable Juices Abou- Raya, M .A. ${ }^{1}$; G. A. Ghoniem ${ }^{1}$; A. K. Abd EL Hameed ${ }^{2}$ and N. M. El rayes ${ }^{2}$ ${ }^{1}$ Food Industries Dept. Fac. of Agric. Mansoura Univ., Egypt. \\ ${ }^{2}$ Department of horticultural crops .Research Department, Food Tech, Giza, Egypt.
}

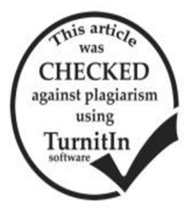

\section{ABSTRACT}

This work was aimed to obtain the best stage of wheat grains germination and determine the sensory quality and acceptability which considered as an important indicator of potential consumer preferences of fresh beverages from wheat grass juice with some fruit and vegetable juices. The data showed that the best result in organoleptic properties of wheat grass beverage in overall acceptability was the second stage (14 days) for germination of sample No. 11(42.06) followed by samples 12, 10, 8 and 7 ( 39.9, 36.32, 35.83 and 34.39 respectively). Developing and characterizing functional drinks of green wheat grass beverage with bananas, guava, lemon and strawberries blends were showed that the sensory evaluation and overall acceptability of the best samples No 10, 2, 8 and 1 were recorded $(47.5,45.13,42.65$ and 40.83 respectively), for wheat grass beverage containing mixture of banana and guava, wheat grass beverage contains banana, wheat grass beverage contains guava and control (wheat grass beverage) sample 1. On the other side, wheat grass beverages containing lemon and strawberries were rejected in all proportions.

\section{INTRODUCTION}

Natural beverages are Healthy beverages with balanced amounts of nutrients and low amounts of sugar, fat and salt. High consumption of fruits and vegetables is associated with a lower risk of coronary heart disease, Alzheimer's disease and prostate cancer (Schurman, et al., 1998; Dai, et al., 2006; Joshipura, et al., 2001). Juices obtained during the fruit age without any pasteurization process are an alternative way to consume fresh fruits and vegetables. In addition, these products cater to the needs of modern consumers, who are increasingly buying ready-to-eat food to save time, without abandoning the fun and food entrees associated with a healthy diet (Endrizzi, et al., 2009). Fruit and vegetable juices have become important in recent years because of the general increase in the consumption of natural juice as an alternative to beverages containing traditional caffeine such as coffee, tea or soft drinks (Kaur, et al., 2009). For maximum benefit of their food, these fresh vegetables should be consumed. Fruit and vegetable juices have become a part of our diets due to enriches minerals, vitamins, low energy value and unique taste (Cassano, et al., 2003), but nowadays cereal grasses are also used as health drink and are becoming popular due to an important sources of antioxidants, bioactive compounds, chlorophylls, amino acids, vitamins and enzymes (Mujoriya, and Bodla, 2011). Wheat grass is a young herb from the normal wheat plant Triticum aestivum Linn., Family Poeaceae (Graminae). Known as "green blood" because of its high content of chlorophyll, this accounts for $70 \%$ of its chemical components (Chauhan, 2014). Wheat grass can be squeezed or dried into powder and used for animal and human consumption, including essential nutrients and vitamins. In the form of fresh juice, contains high concentrations of chlorophyll, active enzymes, vitamins and other nutrients (Mujoriya, and Bodla, 2011).

\section{MATERIALS AND METHODS}

\section{Materials:}

Wheat grains (Triticum aestivum) were grown in the laboratory. The fruits, vegetables such as (guava, bananas, strawberries and lemon) and sugar were purchased from the local market.
Methods:

\section{Growing of wheat grass}

(Wheat Grass) Triticum aestivum can easily grow at home using the following procedures. Single algae combine the feces with three parts that are grown and placed in plastic trays (at least 2 inches).Soak a cup of wheat for 24 hours and then rinse. One cup of seed would be enough for a $25 \times 35 \mathrm{~cm}$ tray. Mix the soil water first, and then evenly distribute the wheat to the wet soil. Cover the wheat grass with a towel cover and place it near the window to ensure appropriate ventilation for three days, and keep away from direct sunlight. In the first three days, in the morning, water like that seed is completely soaked in water. In theevening, lightly spray thewater with a spray bo ttle.On the fifth day, young people are photographed at a height of more than one inch. Now, water only once a day but makes sure the soil is moist to keep the roots moist. Around the ninth or tenth day, the wheatgrass grows to 6-7 inches and it was ready for harvest.

At this point, the grass is at the peak of his food. Cut grass, and extract juice either manually or using fresh juice. Be careful not to plant all wheat grains. Nonenabling grains will encourages mold growth, which can contaminate nearby plants and create allergic effects. (Olguin, A. 2008).

Table 1. Stages of wheat germination:-

\begin{tabular}{lc}
\hline Stages & Time days \\
\hline The first stage & 7 days \\
The second stage & 14 days \\
Third stage & 21 days \\
\hline
\end{tabular}

Preparation of guava, bananas, strawberries, lemon and wheat grass juices:

Wheat grass juice was blended with some fruit and vegetables namely at different proportions guava, bananas, fresh and ripe strawberries and lemon to prepare new beverages formulas. Fruit peeled and crushed in Philips Juicer and mixer (HL1631) to extract juice. Lemon's had been cut and pressed with the hand juicer to extract the juice. The Lemon juice was filtered at the crossing and in a bottle. Wheat grass juice was extracted, also various additions of fruits and vegetables using Philips Juicer and then mixer (HL1631) and got clear juice by passing the gauze cloth. After extracting all juices, a mixture of juices 
and ingredients has been prepared to get beverages formulas.

Organoleptic properties:

All prepared beverages were evaluated by 10 panel testes in Food Technology Research Institute to determine the quality and acceptance for the consumer preferences. All formulas were evaluated for different attributes namely taste, consistency,color,smell,acceptability and overall acceptability using a structured scale from 1 to 6 (Waghray, et al., 2012).

Statistical analysis:

Data collected from the organoleptic properties were statistically analyzed by less significant differences (L.S.D) at $5 \%$ of the probability measures according to Snedecor and Cochran (1980).

\section{RESULTS AND DISCUSSION}

\section{1-Wheat grass in various stages of germination:}

Table (2) showed that fresh juice was prepared from germination of wheat grains from several stages. Three stages were carried out as follows, the first stage 7 days, the second stage 14 days and the third stage was 21 days, whereas different concentrations of sugar (40,50 and $60 \mathrm{~g}$ ) and water (800 and $1000 \mathrm{ml})$ with a fixed weight of wheat grass. Sensory acceptance of fresh tested juices was choosing the best germination stage. The results in Table (3) and fig (1) indicated that the best result in organoleptic properties of wheat grass juice in total admissions was the second stage (14 days) for germination of sample No. 11 (42.06), the juice was composed of $50 \mathrm{~g}$ fresh wheat grass $+50 \mathrm{~g}$ sugar $+1000 \mathrm{ml}$ Water followed by samples No 12 , 10,8 and $7(39.9,36.32,35.83$ and 34.39 respectively, in overall acceptability). The lowest results for samples were $1,2,3,6,4$ and 5, (22.3, 23.6, 23.8, 23.5, 24.41 and 25.5, respectively). These results are in agreement with those Mondal, A. and Saxena, D. (2016). The results showed that the juice of wheat grass and barley grass juice mixed separately with fruit juices was presented to give an acceptable sensory image. All this results will form a more acceptable form of green grass juice and is likely to increase its popularity.

Table 2. Wheat grass blends in various stages of germination with different additives of sugar and water

The first stage(7 days) The second stage(14 days)

(1) $50 \mathrm{~g}$ fresh wheat grass $+40 \mathrm{~g}$ sugar $+800 \mathrm{ml}$ Water.

(2) $50 \mathrm{~g}$ fresh wheat grass $+50 \mathrm{~g}$ sugar $+800 \mathrm{ml}$ Water.

(3) $50 \mathrm{~g}$ fresh wheat grass $+60 \mathrm{~g}$ sugar $+800 \mathrm{ml}$ Water.

(4) $50 \mathrm{~g}$ fresh wheat grass $+40 \mathrm{~g}$ sugar $+1000 \mathrm{ml}$ Water.

(5) $50 \mathrm{~g}$ fresh wheat grass $+50 \mathrm{~g}$ sugar+1000 $\mathrm{ml}$ Water.

(6) $50 \mathrm{~g}$ fresh wheat grass $+60 \mathrm{~g}$ sugar $+1000 \mathrm{ml}$ Water.
(7) $50 \mathrm{~g}$ fresh wheat grass $+40 \mathrm{~g}$ sugar $+800 \mathrm{ml}$ Water.

(8) $50 \mathrm{~g}$ fresh wheat grass $+50 \mathrm{~g}$ sugar $+800 \mathrm{ml}$ Water.

(9) $50 \mathrm{~g}$ fresh wheat grass $+60 \mathrm{~g}$ sugar $+800 \mathrm{ml}$ Water.

(10) $50 \mathrm{~g}$ fresh wheat grass $+40 \mathrm{~g}$ sugar $+1000 \mathrm{ml}$ Water.

(11) $50 \mathrm{~g}$ fresh wheat grass $+50 \mathrm{~g}$ sugar $+1000 \mathrm{ml}$ Water.

(12) $50 \mathrm{~g}$ fresh wheat grass $+60 \mathrm{~g}$ sugar $+1000 \mathrm{ml}$ Water.
Third stage(21 days)

(13) $50 \mathrm{~g}$ fresh wheat grass $+40 \mathrm{~g}$ sugar+800 $\mathrm{ml}$ Water.

(14) $50 \mathrm{~g}$ fresh wheat grass $+50 \mathrm{~g}$ sugar+800 $\mathrm{ml}$ Water.

$(15+) 50 \mathrm{~g}$ fresh wheat grass $+60 \mathrm{~g}$ sugar+800 $\mathrm{ml}$ Water.

(16) $50 \mathrm{~g}$ fresh wheat grass $+40 \mathrm{~g}$ sugar $+1000 \mathrm{ml}$ Water.

(17) $50 \mathrm{~g}$ fresh wheat grass $+50 \mathrm{~g}$ sugar $+1000 \mathrm{ml}$ Water.

(18) $50 \mathrm{~g}$ fresh wheat grass $+60 \mathrm{~g}$ sugar $+1000 \mathrm{ml}$ Water.

Table 3. Organoleptic properties of different states of germination for wheat grass blends

\begin{tabular}{|c|c|c|c|c|c|c|}
\hline $\begin{array}{l}\text { Properties } \\
\text { Samples }\end{array}$ & $\begin{array}{c}\text { Taste } \\
\text { (10) }\end{array}$ & $\begin{array}{l}\text { Color } \\
\text { (10) }\end{array}$ & $\begin{array}{c}\text { Consistency } \\
\text { (10) }\end{array}$ & $\begin{array}{l}\text { Odor } \\
\text { (10) }\end{array}$ & $\begin{array}{c}\text { Palatability } \\
\text { (10) }\end{array}$ & $\begin{array}{c}\text { Overall } \\
\text { Acceptability (50) }\end{array}$ \\
\hline $\begin{array}{l}\text { Sample (1) } \\
\text { control }\end{array}$ & $4 \mathrm{~h} \pm 0.19$ & $5.1 \mathrm{k} \pm 1$ & $4.2 \mathrm{j} \pm 0.19$ & $4 \mathrm{f} \pm 1$ & $5 \mathrm{~g} \pm 0.39$ & $22.3 \mathrm{e} \pm 0.9$ \\
\hline Sample (2) & $4.2 \mathrm{~h} \pm 0.1$ & $5.4 \mathrm{ijk} \pm 0.79$ & $4.6 \mathrm{hij} \pm 0.1$ & $4.2 \mathrm{ef} \pm 0.6$ & $5.2 \mathrm{fg} \pm 0.19$ & $23.6 \mathrm{e} \pm 0.3$ \\
\hline Sample (3) & $4 \mathrm{~h} \pm 0.12$ & 5.5 hijk \pm 0.29 & $5 \mathrm{hi} \pm 0.8$ & 4.5 ef \pm 0.3 & $4.8 \mathrm{~g} \pm 0.2$ & $23.8 \mathrm{e} \pm 0.5$ \\
\hline Sample (4) & $4.3 \mathrm{~h} \pm 0.59$ & $5.2 \mathrm{k} \pm 0.39$ & $5.3 \mathrm{gh} \pm 0.3$ & $4.8 \mathrm{ef} \pm 0.69$ & $4.8 \mathrm{~g} \pm 0.69$ & $24.41 \mathrm{e} \pm 2$ \\
\hline Sample (5) & $4.1 \mathrm{~h} \pm 0.1$ & 5.8 ghijk \pm 0.49 & $5.23 \mathrm{hi} \pm 0.32$ & $5.15 \mathrm{de} \pm 0.15$ & $5.5 \mathrm{efg} \pm 0.5$ & $25.5 \mathrm{e} \pm 0.3$ \\
\hline Sample (6) & $4 \mathrm{~h} \pm 0.5$ & $5.3 \mathrm{jk} \pm 0.19$ & $4.5 \mathrm{ij} \pm 0.5$ & $4.5 \mathrm{ef} \pm 0.5$ & $5.2 \mathrm{fg} \pm 0.2$ & $23.5 \mathrm{e} \pm 1$ \\
\hline Sample (7) & $7.2 \mathrm{bcd} \pm 0.29$ & $7 \mathrm{cde} \pm 1$ & $7.19 \mathrm{~cd} \pm 1.1$ & $6.99 b c \pm 0.99$ & $6 \mathrm{def} \pm 0.3$ & $34.39 \mathrm{~cd} \pm 0.9$ \\
\hline Sample (8) & 7 bcde \pm 1 & $7.2 \mathrm{bcd} \pm 0.2$ & $7.4 b c \pm 0.39$ & $7.02 \mathrm{bc} \pm 1.97$ & $7.2 \mathrm{bc} \pm 0.2$ & $35.83 \mathrm{bc} \pm 1$ \\
\hline Sample (9) & $6.9 \mathrm{cdef} \pm 0.9$ & $6.7 \mathrm{cdef} \pm 0.69$ & $6.8 \mathrm{cde} \pm 0.16$ & $6.7 \mathrm{bc} \pm 0.69$ & $6.8 \mathrm{~cd} \pm 0.8$ & $33.9 \mathrm{~cd} \pm 0.2$ \\
\hline Sample (10) & $7.4 \mathrm{bc} \pm 0.99$ & $7.5 \mathrm{abc} \pm 0.5$ & $7.01 \mathrm{~cd} \pm 0.19$ & $7.11 \mathrm{~b} \pm 0.11$ & $7.3 \mathrm{bc} \pm 0.3$ & $36.32 \mathrm{bc} \pm 0.32$ \\
\hline Sample (11) & $8.3 \mathrm{a} \pm 0.3$ & $8.3 \mathrm{a} \pm 0.19$ & $8.4 \mathrm{a} \pm 0.2$ & $8.5 \mathrm{a} \pm 0.5$ & $8.59 \mathrm{a} \pm 0.6$ & $42.06 \mathrm{a} \pm 0.9$ \\
\hline Sample (12) & $7.8 \mathrm{ab} \pm 0.79$ & $7.99 \mathrm{ab} \pm 0.2$ & $8 a b \pm 1$ & $8.2 \mathrm{a} \pm 0.19$ & $8 a b \pm 1$ & $39.9 \mathrm{ab} \pm 1$ \\
\hline Sample (13) & $6 \mathrm{~g} \pm 0.9$ & 6.4 defgh \pm 0.29 & $6.03 \mathrm{fg} \pm 0.5$ & $6.08 \mathrm{~cd} \pm 0.8$ & $6 \operatorname{def} \pm 1$ & $30.51 \mathrm{~d} \pm 1$ \\
\hline Sample (14) & $6.1 \mathrm{fg} \pm 0.5$ & $6.42 \operatorname{defg} \pm 0.8$ & $6.07 \mathrm{ef} \pm 0.13$ & $6.07 \mathrm{~cd} \pm 0.2$ & $6.1 \mathrm{def} \pm 0.2$ & $30.77 \mathrm{~d} \pm 0.2$ \\
\hline Sample (15) & $6.29 \mathrm{efg} \pm 0.12$ & $6.5 \mathrm{defg} \pm 0.1$ & $6.05 \mathrm{efg} \pm 0.18$ & $6.02 \mathrm{~cd} \pm 0.2$ & $6 \operatorname{def} \pm 1.2$ & $30.79 \mathrm{~d} \pm 1.2$ \\
\hline Sample (16) & $6.15 \mathrm{efg} \pm 0.14$ & 6.19 efghi \pm 0.4 & $6.5 \mathrm{def} \pm 0.2$ & $6.6 \mathrm{bc} \pm 0.2$ & $6.08 \mathrm{def} \pm 0.3$ & $31.53 \mathrm{~cd} \pm 1$ \\
\hline Sample (17) & $6.5 \mathrm{defg} \pm 0.5$ & 6.1 fghij \pm 0.2 & $6.18 \mathrm{ef} \pm 0.17$ & $6.9 \mathrm{bc} \pm 0.9$ & $6.5 \mathrm{~cd} \pm 0.6$ & $32.18 \mathrm{~cd} \pm 0.2$ \\
\hline Sample (18) & $6.2 \mathrm{efg} \pm 0.2$ & 6.19 efghij \pm 1 & $6.15 \mathrm{ef} \pm 0.12$ & $6.1 \mathrm{~cd} \pm 0.2$ & $6.19 \mathrm{de} \pm 0.1$ & $30.84 \mathrm{~d} \pm 0.79$ \\
\hline L.S.D $(\mathrm{P} \geq 0.05)$ & 0.85 & 0.89 & 0.76 & 1 & 0.91 & 4.2 \\
\hline
\end{tabular}




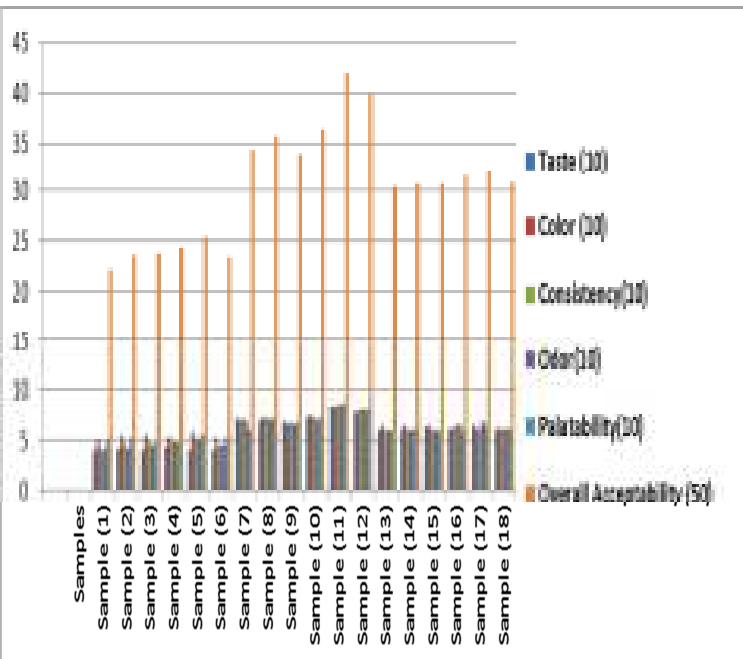

Fig. 1. Organoleptic properties of different states of germination for wheat grass blends.

2- The effect of adding some fruits and vegetables to wheatgrass blends:

Results in Table (4) show that also the best juices obtained for germinated the fresh wheat grass in terms of sensory evaluation was in the second stage of germination various additions of fruits and vegetables were added to this juice such as guava, banana, lemon and strawberry, and then choose the best juices in terms of sensory evaluation.

The results showed that samples $10,2,8$ and 1 were the best in terms of sensory evaluation, consisted of $50 \mathrm{~g}$ fresh wheat grass $+50 \mathrm{~g}$ sugar $+1000 \mathrm{ml}$ Water $+100 \mathrm{~g}$ guava $+100 \mathrm{~g}$ banana $; 50 \mathrm{~g}$ fresh wheat grass $+50 \mathrm{~g}$ sugar $+1000 \mathrm{ml} \mathrm{Water}+200 \mathrm{~g}$ guava $; 50 \mathrm{~g}$ fresh wheat grass +50 $\mathrm{g}$ sugar $+1000 \mathrm{ml}$ Water $+200 \mathrm{~g}$ banana and $50 \mathrm{~g}$ fresh wheat grass $+50 \mathrm{~g}$ sugar $+1000 \mathrm{ml}$ Water $+200 \mathrm{~g}$ guava, respectively. The obtained results are in accordance with those reported by Liana-Claudia, et al., (2016). The juice obtained has a silky sweet taste, a unique flavor and a very nice smell. This product targets all consumer categories and offers a perfect snack in the morning for those who are concerned about a healthy lifestyle.
3- Sensory properties of the best juice prepared from the addition of some fruits and vegetables to wheat grains blends:

The results in Table (5) and Fig (2) show that the sensory evaluation and overall acceptability of the best samples, were No $10,2,8$ and 1 they recorded 47.5, $45.13,42.65$ and 40.83 , respectively of wheat grass beverage contains bananas, wheat grass beverage contains guava, wheat grass beverage contains banana and guava or wheat grass beverage while wheat grass beverage contains lemon and wheat grass beverage contains strawberries were rejected in all its proportions, (samples No 4, 5, 6 and 7, recorded 17.47, 15.23, 19.45 and 15.08 respectively). The obtained results are in accordance with those reported by (Waghray, et al., 2012). They assessment of consumer acceptance by appearance, fragrance, taste and general acceptance of fresh juices. Carrot, wheat grass, bitter gourd juices, and sensory evaluation.

Table 4. The effect of adding some fruits and vegetables to wheatgrass blends

\begin{tabular}{|c|c|}
\hline Samples & Additions \\
\hline (1) & $\begin{array}{c}50 \mathrm{~g} \text { fresh wheat grass }+50 \mathrm{~g} \text { sugar }+1000 \mathrm{ml} \\
\text { Water. }\end{array}$ \\
\hline (2) & $\begin{array}{l}50 \mathrm{~g} \text { fresh wheat grass }+50 \mathrm{~g} \text { sugar }+1000 \mathrm{ml} \\
\text { Water }+200 \mathrm{~g} \text { guava. }\end{array}$ \\
\hline (3) & $\begin{array}{l}50 \mathrm{~g} \text { fresh wheat grass }+50 \mathrm{~g} \text { sugar }+1000 \mathrm{ml} \\
\text { Water }+250 \mathrm{~g} \text { guava. }\end{array}$ \\
\hline (4) & $\begin{array}{c}50 \mathrm{~g} \text { fresh wheat grass }+50 \mathrm{~g} \text { sugar }+1000 \mathrm{ml} \\
\text { Water }+50 \mathrm{ml} \text { lemon juices. }\end{array}$ \\
\hline (5) & $\begin{array}{c}50 \mathrm{~g} \text { fresh wheat grass }+50 \mathrm{~g} \text { sugar }+1000 \mathrm{ml} \\
\text { Water }+100 \mathrm{ml} \text { lemon juices. }\end{array}$ \\
\hline (6) & $\begin{array}{l}50 \mathrm{~g} \text { fresh wheat grass }+50 \mathrm{~g} \text { sugar }+1000 \mathrm{ml} \\
\text { Water }+200 \mathrm{~g} \text { strawberries. }\end{array}$ \\
\hline (7) & $\begin{array}{l}50 \mathrm{~g} \text { fresh wheat grass }+50 \mathrm{~g} \text { sugar }+1000 \mathrm{ml} \\
\text { Water }+250 \mathrm{~g} \text { strawberries. }\end{array}$ \\
\hline (8) & $\begin{array}{l}50 \mathrm{~g} \text { fresh wheat grass }+50 \mathrm{~g} \text { sugar }+1000 \mathrm{ml} \\
\text { Water }+200 \mathrm{~g} \text { banana. }\end{array}$ \\
\hline (9) & $\begin{array}{l}50 \mathrm{~g} \text { fresh wheat grass }+50 \mathrm{~g} \text { sugar }+1000 \mathrm{ml} \\
\text { Water }+250 \mathrm{~g} \text { banana. }\end{array}$ \\
\hline (10) & $\begin{array}{r}50 \mathrm{~g} \text { fresh wheat grass }+50 \mathrm{~g} \text { sugar }+1000 \mathrm{ml} \\
\text { Water }+100 \mathrm{~g} \text { guava }+100 \mathrm{~g} \text { banana. }\end{array}$ \\
\hline (11) & $\begin{array}{r}50 \mathrm{~g} \text { fresh wheat grass }+50 \mathrm{~g} \text { sugar }+1000 \mathrm{ml} \\
\text { Water }+125 \mathrm{~g} \text { guava }+125 \mathrm{~g} \text { banana. }\end{array}$ \\
\hline
\end{tabular}

Table 5. Organoleptic properties for the best juice prepared from adding some fruits and vegetables to wheatgrass blends

\begin{tabular}{|c|c|c|c|c|c|c|}
\hline $\begin{array}{l}\text { Properties } \\
\text { Samples }\end{array}$ & $\begin{array}{l}\text { Taste } \\
\text { (10) }\end{array}$ & $\begin{array}{l}\text { Color } \\
\text { (10) }\end{array}$ & $\begin{array}{c}\text { Consistency } \\
\text { (10) }\end{array}$ & $\begin{array}{l}\text { Odor } \\
\text { (10) }\end{array}$ & $\begin{array}{l}\text { Palatability } \\
\text { (10) }\end{array}$ & $\begin{array}{l}\text { Overall Acceptability } \\
(\mathbf{5 0 )}\end{array}$ \\
\hline Sample (1) & $8.3 \mathrm{abc} \pm 1.7$ & $8.04 b c \pm 1$ & $8.29 \mathrm{~cd} \pm 1$ & $8.2 b c \pm 0.19$ & $8 b \pm 0.5$ & $40.83 \mathrm{c} \pm 2.8$ \\
\hline Sample (2) & $9 \mathrm{ab} \pm 0.3$ & $8.9 \mathrm{a} \pm 0.5$ & $9.13 \mathrm{ab} \pm 0.14$ & $9.09 \mathrm{a} \pm 0.99$ & $9 \mathrm{a} \pm 1$ & $45.13 \mathrm{~b} \pm 0.43$ \\
\hline Sample (3) & $7.8 \mathrm{bc} \pm 1$ & $7.5 \mathrm{~cd} \pm 0.5$ & $7.66 \mathrm{de} \pm 0.16$ & $7.46 \mathrm{~cd} \pm 0.5$ & $7.22 \mathrm{~b} \pm 1.99$ & $37.64 \mathrm{de} \pm 1$ \\
\hline Sample (4) & $3.6 \mathrm{~d} \pm 0.1$ & $3.1 \mathrm{e} \pm 0.3$ & $3.6 \mathrm{fg} \pm 0.4$ & $3.76 \mathrm{ef} \pm 0.75$ & $3.16 \mathrm{~d} \pm 0.16$ & $17.47 \mathrm{fg} \pm 0.8$ \\
\hline Sample (5) & $3.2 \mathrm{~d} \pm 0.3$ & $3.0 \mathrm{e} \pm 0.5$ & $3.01 \mathrm{~g} \pm 0.5$ & $3.02 \mathrm{f} \pm 1$ & $3 \mathrm{~d} \pm 1.1$ & $15.23 \mathrm{f} \pm 0.72$ \\
\hline Sample (6) & $3.8 \mathrm{~d} \pm 0.3$ & $3.6 \mathrm{e} \pm 0.1$ & $3.92 \mathrm{f} \pm 0.29$ & $3.99 \mathrm{e} \pm 0.99$ & $3.81 \mathrm{~d} \pm 0.8$ & $19.45 \mathrm{f} \pm 1.5$ \\
\hline Sample (7) & $3 \mathrm{~d} \pm 0.2$ & $3.1 \mathrm{e} \pm 0.5$ & $3.04 \mathrm{~g} \pm 0.4$ & $3.01 \mathrm{f} \pm 0.1$ & $3 \mathrm{~d} \pm 0.2$ & $15.08 \mathrm{f} \pm 1.2$ \\
\hline Sample (8) & $8.79 \mathrm{ab} \pm 0.6$ & $8.4 \mathrm{ab} \pm 0.3$ & $8.6 \mathrm{bc} \pm 0.19$ & $8.81 \mathrm{ab} \pm 0.29$ & $8.04 \mathrm{~b} \pm 1.2$ & $42.65 \mathrm{c} \pm 1.43$ \\
\hline Sample (9) & $8 \mathrm{bc} \pm 0.5$ & $7.9 \mathrm{bcd} \pm 0.19$ & 7.8 cde \pm 0.59 & $7.66 \mathrm{~cd} \pm 0.8$ & $7.03 \mathrm{c} \pm 1.99$ & $38.39 \mathrm{~d} \pm 0.69$ \\
\hline Sample (10) & $9.5 \mathrm{a} \pm 0.5$ & $9.1 \mathrm{a} \pm 0.3$ & $9.5 \mathrm{a} \pm 0.6$ & $9.6 \mathrm{a} \pm 0.29$ & $9.8 \mathrm{a} \pm 0.6$ & $47.5 \mathrm{a} \pm 0.88$ \\
\hline Sample (11) & $7.3 \mathrm{c} \pm 0.3$ & $7.1 \mathrm{~d} \pm 0.4$ & $7.02 \mathrm{e} \pm 0.5$ & $7 \mathrm{~d} \pm 0.8$ & $7.04 \mathrm{c} \pm 0.3$ & $35.46 \mathrm{e} \pm 1.36$ \\
\hline $\begin{array}{l}\text { L.S.D } \\
(\mathrm{P} \geq 0.05)\end{array}$ & 1.35 & 0.82 & 0.81 & 0.85 & 0.93 & 2.31 \\
\hline
\end{tabular}

Sample (1): $50 \mathrm{~g}$ fresh wheat grass $+50 \mathrm{~g}$ sugar $+1000 \mathrm{ml}$ Water.

Sample (2): $50 \mathrm{~g}$ fresh wheat grass $+\mathbf{5 0} \mathrm{g}$ sugar $+\mathbf{1 0 0 0} \mathrm{ml}$ Water $+\mathbf{2 0 0} \mathrm{g}$ guava.

Sample (3): $50 \mathrm{~g}$ fresh wheat grass $+50 \mathrm{~g}$ sugar $+1000 \mathrm{ml}$ Water $+250 \mathrm{~g}$ guava.

Sample (4) $50 \mathrm{~g}$ fresh wheat grass $+50 \mathrm{~g}$ sugar $+1000 \mathrm{ml}$ Water $+\mathbf{5 0} \mathrm{ml}$ lemon juices.

Sample (5): $50 \mathrm{~g}$ fresh wheat grass $+\mathbf{5 0} \mathrm{g}$ sugar $+1000 \mathrm{ml}$ Water $+100 \mathrm{ml}$ lemon juices.

Sample (6): $50 \mathrm{~g}$ fresh wheat grass $+50 \mathrm{~g}$ sugar $+1000 \mathrm{ml}$ Water $+200 \mathrm{~g}$ strawberries.

Sample (7): $50 \mathrm{~g}$ fresh wheat grass $+\mathbf{5 0} \mathrm{g}$ sugar $+\mathbf{1 0 0 0} \mathrm{ml}$ Water $+\mathbf{2 5 0} \mathrm{g}$ strawberries

Sample (8): $50 \mathrm{~g}$ fresh wheat grass $+50 \mathrm{~g}$ sugar $+1000 \mathrm{ml}$ Water $+200 \mathrm{~g}$ banana.

Sample (9):50 $\mathrm{g}$ fresh wheat grass $+50 \mathrm{~g}$ sugar $+1000 \mathrm{ml}$ Water $+250 \mathrm{~g}$ banana

Sample (10): $50 \mathrm{~g}$ fresh wheat grass $+50 \mathrm{~g}$ sugar $+1000 \mathrm{ml}$ Water $+100 \mathrm{~g}$ guava $+100 \mathrm{~g}$ banana

Sample (11): $50 \mathrm{~g}$ fresh wheat grass $+50 \mathrm{~g}$ sugar $+1000 \mathrm{ml}$ Water $+125 \mathrm{~g}$ guava $+125 \mathrm{~g}$ banana. 


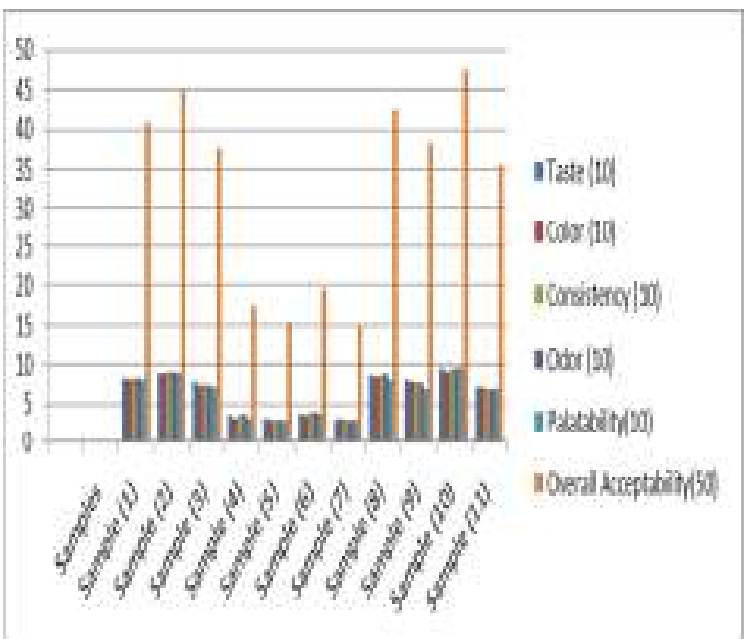

Fig.2. Organoleptic properties for the best juice prepared from adding some fruits and vegetables to wheatgrass blends.

\section{CONCLUSION}

Wheat germination was carried out to obtain wheat grass through 3 stages of germination first stage after 7 days second stage after 14 days while third stage after 21 days and they used wheat grass juice production the best results in the sensory characteristics of wheat juice General Acceptance is was the second stage (14 days) of germination production (sample No. 11). Develop and characterize functional drinks of green wheat juice with banana, guava, lemon juice and strawberries. Wheat grass beverage contains banana and guava or wheat grass beverage contains banana, or wheat grass beverage contains guava showed the best of beverages. While the wheat grass beverage contains lemon and wheat grass beverage contains strawberry were rejected in all proportions.

\section{REFERENCES}

Cassano, A;Drioli, E; Galaverna, G; Marchelli, R; Di Silvestro, G; and Cagnasso, P. (2003).Clarification and concentration of citrus and carrot juices by integrated membrane processes, J. Food Eng, 57:153-163.

Chauhan, M. (2014). A pilot study on wheat grass juice for its phytochemical, nutritional and therapeutic potential on chronic diseases. International Journal of Chemical Studies, 2(4): 27-34.
Dai, Q; Borenstein, A.R; Wu, Y; Jackson, J.C. and Larson, E.B. (2006).Fruit and vegetable juices and Alzheimer's disease: The Kame Project. Am J Med, 119: 751-759.

Endrizzi, I; Pirretti, G; Calo, D.G and Gasperi, F.(2009). A consumer study of fresh juices containing berry fruits. Journal of the Science of Food and Agriculture, 89(7): 1227-1235.

Joshipura, KJ; Hu FB and Willett, W.C. (2001). The effect of fruit and vegetable intake on risk for coronary heart disease. Ann Intern Med, 134: 1106-1114.

Kaur, S; Sarkar, B.C; Sharma, H.K and Singh,C.(2009). Response surface optimization of conditions for the clarification of guava fruit juice using commercial enzyme. J Food Pro Eng, 34(4): 1298-1318.

Liana-Claudia, S; Tofană, M; Domokos, B; Socaci, S.A; Pop, C. R. and Fărcaș, A. C. (2016).Development of functional beverage from wheat grass juice. Bulletin UASVM Food Science and Technology, 73(2).

Mondal, A. and Saxena, D. (2016).Development of barley grass juice and wheat grass juice as functional food with added other fruit juices and their organoleptic evaluation. American International Journal of Research in Formal, Applied \& Natural Sciences, 16(1): 17-22.

Mujoriya, R. and Bodla, R.B. (2011). A study on wheat grass and its nutritional value. Food Science and Quality Management, 2:1-9.

Olguin, A. (2008). The new way to grow wheatgrass: Using bio technology to grow wheatgrass with the highest nutrients. Version 41. Knol. Accessed from $<\mathrm{http}$ ://knol.google.com/k/andrea-olguin/the-newway-to-grow-wheatgrass/1sitjsg5s $3015 / 2>$.

Schuurman, A.G; Goldbohm, R.A; Dorant, E. and Van Den Brandt,P.A. (1998). Vegetable and fruit consumption and prostate cancer risk, a cohort study in the Netherlands. Cancer Epidem Biomark, 7: 673-680.

Snedecor, G. W. and Cochran, W. G. (1980). Statistical methods. Oxford and J. B. H publishing Co. $7^{\text {th }}$ edition.

URL:htpp://www.welikitraw.com.

Waghray, K; Gulla, S; Kumar Santhosh, C; Kumar Praveen, M. and Kumar Akshay, A. (2012). Sensory quality and acceptability of fresh juices. Stud Home Com Sci, 6(3):179-181.

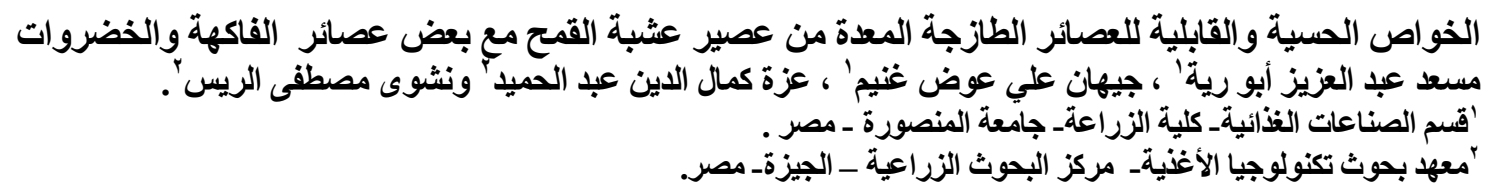

يهدف هذا البحث إلى الحصول على أحسن مرحلة إنبات لحبوب القمح للحصول على عثبة القمح و تقدير الخواص الحسية والقابلية

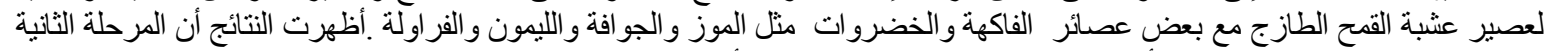

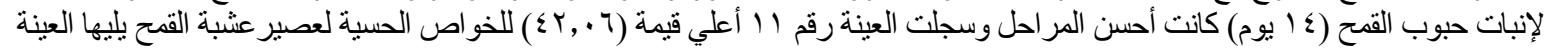

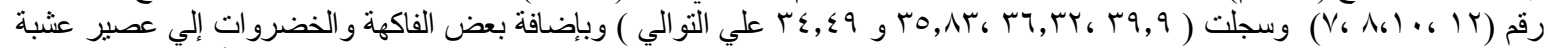

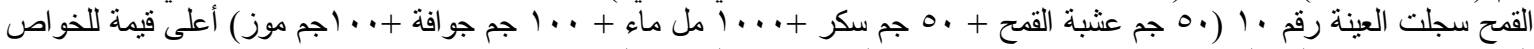

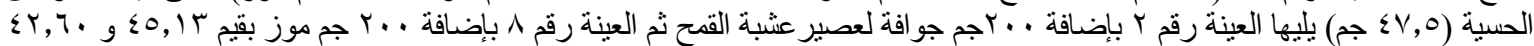

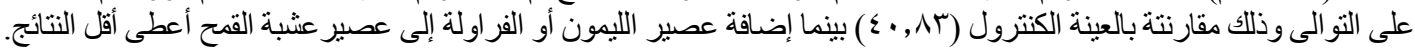

